\title{
Probióticos en dispepsia, microbiota y estreñimiento, gluten en el síndrome de intestino irritable y cannabinoides en trastornos motores y funcionales gastrointestinales
}

Max Schmulson ${ }^{1 *}$ y Miguel Saps ${ }^{2}$

${ }^{1}$ Profesor titular de Medicina, Laboratorio de Higado, Páncreas y Motilidad (HIPAM) - Unidad de Investigación en Medicina Experimental, Facultad de

Medicina, Universidad Nacional Autónoma de México (UNAM), Ciudad de México, México; ${ }^{2}$ Department of Pediatrics, Division of Pediatric Gastroenterology,

Hepatology and Nutrition, University of Miami-Miller School of Medicine, Miami, Fla., EE.UU.

En el tercer número de NeuroGastroLatam Reviews (NGLR) del 2020 se publican cuatro artículos diversos: el uso de probióticos en dispepsia funcional (DF), la microbiota y su modulación en estreñimiento crónico, trastornos del gluten en el síndrome de intestino irritable (SII) y, para finalizar, la relación de los cannabinoides con trastornos motores y funcionales gastrointestinales.

El primer artículo (Surdea-Blaga, Chira y Dumitrascu de Napoca-Rumania) revisa las evidencias sobre el uso de probióticos en $\mathrm{DF}^{1}$. Este es un tópico interesante, ya que los probióticos han sido utilizados y valorados principalmente en pacientes con trastornos intestinales de la interacción intestino-cerebro
$(\mathrm{TIIC})^{2,3}$; pero en los últimos años también ha tomado auge el papel de la microbiota en el estómago y por lo tanto en la fisiopatología y tratamiento de la $\mathrm{DF}^{4}$. Si bien Helicobacter pylori es el microorganismo principalmente comprometido en las patologías del estómago, otras bacterias como los estreptococos se han relacionado con el cáncer gástrico y la gastropatía autoinmune, todos estos trastornos orgánicos $^{5}$. Por su parte, DF, el TIIC por excelencia, que compromete el estómago y el duodeno, también presentan una alteración del eje microbiota-cerebro intestino ${ }^{4}$. Es así como el H. pylori mismo, se ha relacionado con alteraciones de la motilidad gastrointestinal y la sensibilidad visceral en un subgrupo de pacientes con $\mathrm{DF}^{6}$, pero también se ha

\footnotetext{
Correspondencia:

*Max Schmulson-Wasserman

Laboratorio de Hígado, Páncreas y Motilidad (HIPAM)

Unidad de Investigación en Medicina Experimental

Facultad de Medicina

Universidad Nacional Autónoma de México

Hospital General de México

Dr. Balmis, 148

Col. Doctores

C.P. 06726, Ciudad de México, México

E-mail:maxjulio@prodigy.net.mx
}

NeuroGastroLatam Rev. 2020;4:127-132

Received in original form: 14-12-2020

Accepted in final form: 15-12-2020

DOI: 10.24875/NGL.M20000018

2462-7011/@ 2020 Sociedad Latinoamericana de Neurogastroenterología, A.C. Published by Permanyer. This is an open access article under the CC BY-NC-ND license (http://creativecommons.org/licenses/by-nc-nd/4.0/). 
reportado un incremento en la abundancia relativa de Streptococcus en la mucosa duodenal y una reducción de anaeróbicos como Prevotella, Veillonella y Actinomyces ${ }^{7}$. Así mismo, un grupo de pacientes desarrolló DF posterior a una infección gastrointestinal. Una revisión sistemática y metaanálisis determinó la prevalencia de DF Postinfección del 9.55\% sobre 9,517 adultos con gastroenteritis infecciosa con una razón de momios (RM) para presentar DF a los 6 meses de la infección de 2.54 (intervalo de confianza del 95\% [IC 95\%]: 1.76-3.65) en comparación con la prevalencia en la misma población general, si bien los estudios presentaron heterogeneidad significativa $^{8}$. Los patógenos asociados fueron Salmonella spp., Escherichia coli O157, Campylobacter jejuni, Giardia lamblia y Norovirus $^{8}$. Estas alteraciones apuntan entonces a la posibilidad de modular la microbiota gastroduodenal como una de las aproximaciones terapéuticas en estos pacientes. En su artículo, Surdea-Blaga, Chira y Dumitrascu revisan las evidencias sobre el uso terapéutico de probióticos en $\mathrm{DF}^{1}$. A pesar de que las evidencias son escasas, algunas cepas de Lactobacillus y Bifidobacterium han mostrado alguna efectividad en estos pacientes, principalmente en yogures $\mathrm{u}$ otras preparaciones lácteas con estos probióticos, por lo cual los autores sugieren que un ciclo de tratamiento con estos agentes se debe recomendar como tratamiento coadyuvante en pacientes con $\mathrm{DF}^{1}$.

Siguiendo con el tópico de la microbiota, Hanna-Jairala y Bustos-Fernández hacen una revisión sobre la microbiota y su modulación en estreñimiento crónico ${ }^{9}$. Si bien la disbiosis se ha implicado en la fisiopatología de los TIIC bajos como mencionamos anteriormente, el papel exacto de estas alteraciones en la fisiopatología en trastornos como el estreñimiento crónico y/o en SII con estreñimiento no está claro aún. Sin embargo, como lo muestran los autores, hasta el 2019, los estudios sobre microbiota predominaban en pacientes con diarrea que con estreñimiento en cinco veces $^{9}$. No obstante, la revisión de Ohkusa, et al. concluyó que los pacientes con SII con estreñimiento (SII-E) presentan menores niveles de Actinobacteria, incluyendo Bifidobacteria, en muestras fecales y mayores niveles de Bacteroidetes en la mucosa ${ }^{10}$. A pesar de estas evidencias, Hanna-Jairala y Bustos-Fernández consideran que no se puede establecer una firma microbiana propia de los pacientes con estreñimiento. Además, resaltan el papel de las archaeas, específicamente Methanobrevibacter smithii. En su experiencia en pruebas de aliento con aire espirado, los autores han confirmado la mayor concentración de metano en pacientes con estreñimiento y su relación con la gravedad de este ${ }^{11,12}$; lo que les permite concluir que estos microorganismos son un componente determinante de la microbiota en pacientes con estreñimiento, como ha sido descrito por otros ${ }^{13}$. En cuanto a la modulación de la microbiota en pacientes con estreñimiento, los autores afirman que el uso de Psyllium y lactulosa produce cambios en la composición de la microbiota intestinal. La inulina, la lactulosa y el lactilol han mostrado mejorar la frecuencia y consistencia de las heces y disminuir el tiempo del tránsito intestinal en estreñimiento crónico. La rifaximina ha mostrado eficacia en SII-E sola o en combinación con neomicina; esta última además disminuye el tránsito intestinal en estreñimiento crónico. En cuanto a los probióticos, Bifidobacterium lactis DN-173010, B. lactis HN019, Lactobacillus reuteri y Lactobacillus casei 
Shirota, han mostrado beneficios en SII-E'. Hay que mencionar también el papel de probióticos a base de levaduras, como Saccharomyces cerevisiae CNCM I-3856, que ha mostrado mejorar la distensión subjetiva y el dolor abdominal en pacientes con SII-E ${ }^{14}$. Finalmente, en cuanto al transplante de microbiota fecal, las revisiones sistemáticas no han demostrado efectividad en SII en general ${ }^{15-17}$, aunque recientemente se sugiere que el uso de un «super donador» puede producir mejoría en la severidad de los síntomas ${ }^{18}$, pero hasta el momento no hay evidencias específicamente en pacientes con SII-E. Y en cuanto al estreñimiento crónico, quizá las evidencias son más prometedoras sobre la mejoría en frecuencia y consistencia de las evacuaciones, sensación de evacuación incompleta e incluso en la disminución del tránsito intestinal ${ }^{19,20}$.

El tercer artículo de este número de NGLR sigue en perfecta secuencia a los dos anteriores. Esta revisión se refiere al complejo clínico sobre enfermedad celíaca (EC), SII y sensibilidad al gluten no celíaca (SGNC), tres patologías difíciles de diferenciar entre sí, pero que además pueden coexistir, en especial el SII con la EC o con la SGNC, y que se asocian con alteración en la microbiota intestinal ${ }^{21}$. Potent y Olano realizaron una revisión bibliográfica en PubMed y Lilacs de los últimos cinco años, encontrando para nuestra sorpresa solo seis artículos sobre la sobreposición de EC en SII, solo uno sobre SGNC en SII y ninguno sobre la prevalencia de SII en $\mathrm{EC}^{21}$, a pesar de la frecuente sobreposición que encontramos en la clínica y que nos impone un reto diagnóstico y terapéutico (comunicación personal del Dr. Max Schmulson). Además, solo la mitad de los estudios de EC utilizaron el diagnóstico histológico. La prevalencia de EC en SII varió del 2 al 10\% y cuando se utilizó la histología, bajo al rango de 0.8 a $3.3 \%^{21}$.

En realidad, en cuanto a la SGNC falta mucho por conocer. No solo se caracteriza por síntomas gastrointestinales, sino extraintestinales también, lo cual es muy semejante a lo que ocurre en TIIC como el SII ${ }^{22}$. Y si bien una alta proporción de pacientes con SII reporta síntomas asociados con el gluten y por iniciativa propia suspenden el gluten de la dieta, refiriendo mejoría de sus síntomas y calidad de vida $^{23}$, en realidad se desconoce si el gluten por sí solo o algún otro componente proteico del trigo es el responsable de producir los síntomas en estos pacientes ${ }^{22}$. Por otra parte, hay que considerar que un importante grupo de pacientes que refieren sensibilidad al gluten, en realidad presentan sensibilidad a oligosacáridos, disacáridos, monosacáridos y polioles fermentables (FODMAP), entre los cuales los fructanos son los carbohidratos principales en el trigo, y de allí su confusión. Sin embargo, ambas dietas han sido utilizadas como primera medida de tratamiento por muchos especialistas encargados del manejo de pacientes con SII; no obstante, la dieta libre de gluten es mucho más fácil de implementar y menos restrictiva que la dieta baja en FODMAP $^{24}$. Así mismo, si bien la dieta libre de gluten es esencial en el tratamiento de EC, incluso en el manejo de manifestaciones como la dermatitis herpetiforme y alteraciones psicológicas en estos pacientes, no hay suficientes evidencias para su utilización en otros padecimientos como la SGNC o el SII mis$\mathrm{mo}^{25}$. A pesar de lo anterior, en un estudio en Canadá en pacientes con SII-Roma III, no seleccionados, aquellos que reportaron 
sensibilidad al gluten y que tenían antigliadinas IgA positivas respondieron en mayor proporción y de manera significativa a la dieta baja en gluten en comparación con los pacientes sin la presencia de antigliadinas IgA e IgG $(75 \text { vs. 38\% })^{26}$. Además, una mayor proporción de pacientes con estos biomarcadores mejoraron el tránsito intestinal. Más aún, la presencia de antigliadinas IgG fue un marcador predictor de la disminución de síntomas con la dieta libre de gluten en comparación con los pacientes sin este anticuerpo (RM: 128.9; IC 95\%: 1.16-1,427.8; $\mathrm{p}=0.04)^{26}$.

Incluso desde el punto de vista de la fisiopatología del SII, falta mucho por conocer con respecto a la dieta. Por ejemplo, en un estudio que analizó el efecto de la dieta libre de gluten o baja en FODMAP sobre la barrera epitelial de pacientes con SII, solo la segunda mostró mejoría sobre los síntomas y sobre la barrera epitelial del colon, no así la dieta baja en gluten ${ }^{27}$. Incluso cuando se analizó la relación de los haplotipos DQ2 y DQ8 (que son marcadores de EC, pero que pueden estar presentes en pacientes con SGNC) en pacientes con SII y linfocitosis intraduodenal, se determinó que los pacientes con linfocitosis tenían mayor expresión de marcadores proinflamatorios en comparación con los pacientes con SII sin linfocitosis intraduodenal ${ }^{28}$. Sin embargo, la expresión de los marcadores inflamatorios no dependía de la presencia del HLA DQ2 y DQ8 ${ }^{28}$.

Todo lo anterior nos indica que falta mucho por conocer en la relación fisiopatológica del gluten en la EC y la SGNC presentes o asociadas con el SII, y como desencadenantes de los síntomas en estos pacientes. Mientras la literatura evoluciona $y$ estos aspectos se clarifican, Potent y Olano nos presentan un práctico algoritmo que puede ser de utilidad en la clínica ${ }^{21}$. Si el paciente presenta síntomas horas a semanas luego del consumo de gluten $\mathrm{y}$ tiene anticuerpos positivos para EC, es muy probable que se trate de este trastorno. Ante la ausencia de anticuerpos, estaríamos ante un SGNC, un SII o una EC seronegativa, que es poco frecuente. Adicionalmente, si no hay anticuerpos y el paciente presenta otros tipos de atopias o alergias, y los síntomas se presentan de minutos a horas luego del consumo de trigo, podría tratarse de una alergia al gluten o a algún otro componente del trigo ${ }^{21}$.

El último artículo de esta serie (Arboleda, et al.) es una revisión sobre los cannabinoides involucrados en los mecanismos desencadenantes de trastornos motores y funcionales gastrointestinales y en el tratamiento de estos $^{29}$. El artículo analiza la proporción de pacientes que utilizan cannabinoides sin una guía especializada, describe los sistemas endocannabinoides y la presencia de receptores CB1 en el tracto gastrointestinal, las diferencias entre el delta-9-tetrahidrocannabinol (THC) y el cannabidiol (CBD) como fuentes terapéuticas, los medicamentos cannabinoides actualmente disponibles en el mercado, las evidencias científicas y la experiencia clínica para su uso, así como las recomendaciones para utilizar estos agentes en la clínica. En la segunda parte del artículo, se revisa la relación de los cannabinoides con la motilidad del tubo digestivo y con los trastornos motores y funcionales, haciendo énfasis en la hiperemésis por cannabinoides, uno de los nuevos TIIC incluidos en Roma IV ${ }^{29}$. Hay que destacar que la mayoría de los países de Latinoamérica que han aprobado los cannabinoides para uso medicinal, han autorizado el uso de extractos 
orales derivados de la marihuana, también denominada cannabis medicinal. Sin embargo, entre los cuatro productos denominados cannabinoides medicinal o de prescripción o farmacéuticos, los nabiximoles han sido aprobados en más de 30 países, incluyendo Chile, Colombia y Brasil para trastornos espásticos y esclerosis múltiple ${ }^{30}$.

Específicamente en cuanto a los trastornos motores y funcionales gastrointestinales, los cannabinoides utilizados por periodos cortos en estudios mecanísticos, sugieren que pueden atenuar el vaciamiento gástrico y retardar la motilidad colónica, pero parecen tener menor efecto sobre los umbrales sensoriales del intestino $^{31}$. Lo anterior está probablemente mediado por los receptores CB1 que se encuentran en las células epiteliales intestinales, como mencionan Arboleda, et al. ${ }^{29}$. Con respecto al vaciamiento gástrico, por ejemplo, un estudio que comparó los niveles circulantes de endocannabinoides y ácidos grasos derivados de cannabimiméticos en pacientes con gastroparesia y controles reportó menores niveles de los endocannabinoides anandamida y del 2-araquidonil glicerol en mujeres con gastroparesia diabética en comparación con diabéticas sin gastroparesia ${ }^{32}$. Otros monoacilgliceroles como el 2-palmitoil glicerol y el 2-oleoil glicerol también se encontraron disminuidos en estas pacientes con gastroparesia, pero no en hombres. Los autores concluyeron que como la gastroparesia diabética es más común en mujeres y considerando que los cannabinoides son antieméticos, los menores niveles de estos compuestos pueden contribuir a la generación de síntomas como vómito en estas pacientes ${ }^{32}$. Lo anterior nos lleva a la hiperemésis por cannabinoides, un trastornos considerado previamente huérfano, que si bien su etiología está relacionada con los cannabinoides, ha sido considerado por Roma IV un TIIC gastroduodenal debido a que su generación se debe a una alteración del eje intestino-cerebro ${ }^{33}$. Los cannabinoides activan los receptores cannabinoides que inhiben la liberación de transmisores de neuronas presinápticas, a su vez inhibiendo la alfa diacilglicerol lipasa, que previene la síntesis de araquidonil glicerol endocannabinoides-2, con lo cual los cannabinoides pueden desencadenar hiperemésis ${ }^{34}$. En la clínica debemos diferenciar la hiperemésis por cannabinoides del uso de cannabinoides para calmar las náuseas y el vómito en los pacientes con síndrome de vómito cíclico.

En cuanto a los cannabinoides en diarrea, la nabilona (un agonista de receptores CB1) utilizada por tres meses ha mostrado en una serie de casos mejorar el estado de salud en todos los pacientes, disminuir la diarrea y aumentar de peso $^{35}$. En modelos animales, los agonistas de CB1 han demostrado disminuir la hipermotilidad intestinal en ratones ${ }^{36}$, y los agonistas CB2 han demostrado corregir la hipermotilidad colónica inducida por estrés en ratas ${ }^{37}$. Queda mucho por investigar en el efecto de los sistemas endocannabinoides en trastornos motores y TIIC además del efecto terapéutico derivado de la modulación de estos.

Con lo anterior finalizamos el año 2020 en NGLR, un año que quedará marcado en la historia de la humanidad por la pandemia de la enfermedad por coronavirus 2019 (COVID-19), que afectó la vida como la conocemos, nuestras esperanzas, trabajo, la investigación y la productividad. Sin embargo, parece visualizarse la luz al final del túnel con el desarrollo de vacunas que empiezan a distribuirse y a aplicarse. 


\section{BIBLIOGRAFÍA}

1. Surdea-Blaga T, Chira A, Dumitrascu DL. Treatment with probiotics in functional dyspepsia. NeuroGastroLatam Rev. 2020;4:1-11.

2. Ford AC, Harris LA, Lacy BE, Quigley EMM, Moayyedi P. Systematic review with meta-analysis: the efficacy of prebiotics, probiotics, synbiotics and antibiotics in irritable bowel syndrome. Aliment Pharmacol Ther. 2018;48:1044-60.

3. Li B, Liang L, Deng H, Guo J, Shu H, Zhang L. Efficacy and safety of probiotics in irritable bowel syndrome: A systematic review and meta-analysis. Front Pharmacol. 2020;11:332.

4. Ding JH, Jin Z, Yang XX, Lou J, Shan WX, Hu YX, et al. Role of gut microbiota via the gut-liver-brain axis in digestive diseases. World J Gastroenterol. 2020;26:6141-62

5. Conti L, Annibale B, Lahner E. Autoimmune gastritis and gastric microbiota. Microorganisms. 2020;8(11):1827.

6. Suzuki H, Moayyedi P. Helicobacter pylori infection in functional dyspepsia. Nat Rev Gastroenterol Hepatol. 2013;10:168-74.

7. Zhong L, Shanahan ER, Raj A, Koloski NA, Fletcher L, Morrison M, et al. Dyspepsia and the microbiome: time to focus on the small intestine. Gut. 2017;66:1168-9.

8. Futagami S, Itoh T, Sakamoto C. Systematic review with meta-analysis: postinfectious functional dyspepsia. Aliment Pharmacol Ther. 2015;41:177-88.

9. Hanna-Jairala I, Bustos-Fernández LM. NeuroGastroLatam Rev. 2020;4:144-58.

10. Ohkusa T, Koido S, Nishikawa Y, Sato N. Gut microbiota and chronic constipation: A review and update. Front Med (Lausanne). 2019;6:19.

11. Pichetshote N, Rezaie A. Breath tests for functional gastrointestinal disorders: When and for what? NeuroGastroLatam Rev. 2018;2:87-97.

12. Chatterjee S, Park S, Low K, Kong Y, Pimentel M. The degree of breath methane production in IBS correlates with the severity of constipation. Am J Gastroenterol. 2007;102:837-41

13. Ghoshal U, Shukla R, Srivastava D, Ghoshal UC. Irritable bowel syndrome, particularly the constipation-predominant form, involves an increase in Methanobrevibacter smithii, which is associated with higher methane production. Gut Liver. 2016;10:932-8.

14. Spiller R, Pelerin F, Cayzeele Decherf A, Maudet C, Housez B, Cazaubiel M, et al. Randomized double blind placebo-controlled trial of Saccharomyces cerevisiae CNCM I-3856 in irritable bowel syndrome: improvement in abdominal pain and bloating in those with predominant constipation. United European Gastroenterol J. 2016;4:353-62.

15. Ianiro G, Eusebi LH, Black CJ, Gasbarrini A, Cammarota G, Ford AC. Systematic review with meta-analysis: efficacy of faecal microbiota transplantation for the treatment of irritable bowel syndrome. Aliment Pharmacol Ther. 2019;50:240-8.

16. Xu D, Chen VL, Steiner CA, Berinstein JA, Eswaran S, Waljee AK, et al. Efficacy of fecal microbiota transplantation in irritable bowel syndrome: A systematic review and meta-analysis. Am J Gastroenterol. 2019;114:1043-50.

17. Myneedu K, Deoker A, Schmulson MJ, Bashashati M. Fecal microbiota transplantation in irritable bowel syndrome: A systematic review and metaanalysis. United European Gastroenterol J. 2019;7:1033-41.

18. El-Salhy M, Hatlebakk JG, Gilja OH, Bråthen Kristoffersen A, Hausken T. Efficacy of faecal microbiota transplantation for patients with irritable bowel syndrome in a randomised, double-blind, placebo-controlled study. Gut. 2020;69:859-67.

19. Tian H, Ge X, Nie Y, Yang L, Ding C, McFarland LV, et al. Fecal microbiota transplantation in patients with slow-transit constipation: A randomized, clinical trial. PLoS One. 2017;12:e171308.

20. Ding C, Fan W, Gu L, Tian H, Ge X, Gong J,et al. Outcomes and prognostic factors of fecal microbiota transplantation in patients with slow transit constipation: results from a prospective study with long-term follow-up. Gastroenterol Rep (Oxf). 2018;6:101-7.
21. Pontet $Y$, Olano C. Intentando separar la paja del trigo: superposición entre síndrome de intestino irritable, enfermedad celíaca y sensibilidad al gluten no celíaca. NeuroGastroLatam Rev. 2020;4:159-70.

22. Barbaro MR, Cremon C, Wrona D, Fuschi D, Marasco G, Stanghellini V, et al. Non-celiac gluten sensitivity in the context of functional gastrointestinal disorders. Nutrients. 2020;12(12):E3735.

23. Singh P, Nee J. Role of diet in diarrhea-predominant irritable bowel syndrome. J Clin Gastroenterol. 2021;55:25-9.

24. Bellini M, Tonarelli S, Mumolo MG, Bronzini F, Pancetti A, Bertani L, et al Low fermentable oligo- di- and mono-saccharides and polyols (FODMAPs) or gluten free diet: What is best for irritable bowel syndrome? Nutrients. 2020;12(11):3368.

25. Di Liberto D, Carlisi D, D'Anneo A, Emanuele S, Giuliano M, De Blasio A et al. Gluten free diet for the management of non celiac diseases: The two sides of the coin. Healthcare (Basel). 2020;8(4):400.

26. Pinto-Sanchez MI, Nardelli A, Borojevic R, De Palma G, Causada Calo N, McCarville J, et al. Gluten-free diet reduces symptoms, particularly diarrhea, in patients with irritable bowel syndrome and antigliadin IgG. Clin Gastroenterol Hepatol. 2020 Aug 19;S1542-3565(20)31149-6. doi: 10.1016/j. cgh.2020.08.040. Online ahead of print.

27. Ajamian M, Rosella G, Newnham ED, Biesiekierski JR, Muir JG, Gibson PR. Effect of gluten ingestion and FODMAP restriction on intestinal epithelial integrity in patients with irritable bowel syndrome and self-reported noncoeliac gluten sensitivity. Mol Nutr Food Res. 2020 Sep 9;e1901275. doi 10.1002/mnfr.201901275. Online ahead of print.

28. Losurdo G, Todeschini A, Giorgio F, Piscitelli G, Giangaspero A, Ierardi E, et al. Human leukocyte antigen (HLA) haplotype does not influence the inflammatory pattern of duodenal lymphocytosis linked to irritable bowel syndrome. Medicina (Kaunas). 2020;56(12):E660.

29. Arboleda MF, Prosk E, Watier A, et al. Cannabinoids in disorders of gutbrain interaction and gastrointestinal motility. NeuroGastroLatam Rev. 2020;4:171-86.

30. Yadav V, Bever C Jr, Bowen J, Bowling A, Weinstock-Guttman B, Cameron M, et al. Summary of evidence-based guideline: complementary and alternative medicine in multiple sclerosis: report of the guideline development subcommittee of the American Academy of Neurology. Neurology. 2014;82:1083-92.

31. Nasser Y, Woo M, Andrews CN. Cannabis in gastroenterology: Watch your head! A review of use in inflammatory bowel disease, functional gut disorders, and gut-related adverse effects. Curr Treat Options Gastroenterol 2020:1-12.

32. Bashashati M, Leishman E, Bradshaw H, Sigaroodi S, Tatro E, Bright T, et al Plasma endocannabinoids and cannabimimetic fatty acid derivatives are altered in gastroparesis: A sex- and subtype-dependent observation. Neurogastroenterol Motil. 2020 Aug 10;e13961. doi: 10.1111/nmo.13961. Online ahead of print.

33. Schmulson MJ, Drossman DA. What is new in Rome IV. J Neurogastroenterol Motil. 2017;23:151-63.

34. Maselli DB, Camilleri M. Pharmacology, clinical effects, and therapeutic potential of cannabinoids for gastrointestinal and liver diseases. Clin Gastroenterol Hepatol. 2020 Jul 13:S1542-3565(20)30504-8. doi: 10.1016/j. cgh.2020.04.020. Online ahead of print.

35. Pellesi L, Verga MC, De Maria N, Villa E, Pini LA, Guerzoni S. Nabilone administration in refractory chronic diarrhea: a case series. BMC Gastroenterol. 2019;19:105.

36. Salaga M, Binienda A, Tichkule RB, Thakur GA, Makriyannis A, Storr M et al. The novel peripherally active cannabinoid type 1 and serotonin type 3 receptor agonist AM9405 inhibits gastrointestinal motility and reduces abdominal pain in mouse models mimicking irritable bowel syndrome. Eur J Pharmacol. 2018;836:34-43.

37. Lin M, Chen L, Xiao Y, Yu B. Activation of cannabinoid 2 receptor relieves colonic hypermotility in a rat model of irritable bowel syndrome. 2019;31:e13555. 